\title{
Laparoscopic resection of a sigmoid colon lipoma in a young female patient: A case report and review of the literature
}

\author{
GEORGIOS I. PANAGIOTAKIS ${ }^{1 *}$, ALEXANDROS G. ANDREOU ${ }^{1 *}$, IOANNIS E. PETRAKIS ${ }^{1}$, MARIA TZARDI $^{2}$, \\ MARIA DASKALOGIANNAKI ${ }^{3}$ and GEORGIOS E. CHALKIADAKIS ${ }^{1}$
}

Departments of ${ }^{1}$ General Surgery, ${ }^{2}$ Histopathology and ${ }^{3}$ Computed Tomography, University Hospital of Heraklion, Faculty of Medicine, Heraklion 71110, Crete, Greece

Received October 2, 2015; Accepted May 16, 2016

DOI: $10.3892 / \mathrm{ol} .2017 .5594$

\begin{abstract}
Lipomas of the sigmoid colon are rare entities. The present case describes a 27-year-old Caucasian woman who underwent a laparoscopic sigmoidectomy following the detection of a giant lipoma. The young patient was referred to the Emergency Department of the University Hospital of Heraklion (Crete, Greece) in May 2013 after experiencing intermittent abdominal cramping during defecation, and altering episodes of diarrhea and constipation. In addition, the patient described the protrusion of a solid tissue mass shaped like a 'champagne bottle cork' from the anus following defecation. These symptoms had been present for 1 month prior to referral. Physical examination was unremarkable. An urgent colonoscopy revealed a polypoid lesion measuring $2.5 \mathrm{~cm}$ in diameter in the sigmoid colon, which was located $\sim 12 \mathrm{~cm}$ above the anal ring, with a smooth surface and tissue fragility. Tissue samples were obtained and sent for histopathological analysis. Preoperative contrast-enhanced computed tomography was performed urgently and confirmed the presence of a solid mass in the sigmoid colon without enlargement of regional lymph nodes. Following adequate preparation, the patient underwent a laparoscopic sigmoidectomy with intracorporeal termino-terminal colorectal anastomosis, with the use of a circular stapler. The patient had a positive post-operative outcome without complications and was discharged on day 4 post-surgery in an optimal condition. Histopathological examination of the surgical specimen demonstrated a pedunculated lipoma shaped like a 'champagne bottle cork'. The tumor consisted of mature adipose cells. The overlying colonic mucosa showed hyperplastic crypts with regenerative changes. In the lamina propria mild inflammatory infiltration was observed. At
\end{abstract}

Correspondence to: Dr Georgios I. Panagiotakis, Department of General Surgery, University Hospital of Heraklion, Faculty of Medicine, Building C 2nd Floor, Voutes, Heraklion 71110, Crete, Greece

E-mail: gpanagiotakis@hotmail.com

*Contributed equally

Key words: sigmoid colon lipoma, laparoscopic sigmoidectomy, termino-terminal intracorporeal anastomosis
2 years post-surgery, the patient remains asymptomatic without any clinical evidence of recurrence.

\section{Introduction}

Lipomas of the colon are extremely uncommon benign tumors, with a frequency of $0.035-4.4 \%$ worldwide (1). Approximately $90 \%$ of cases originate from the submucosa, while $10 \%$ arise from the subserosa (1). Lipomas have a benign clinical course with a low recurrence rate following surgical excision and the survival rate is $\sim 99 \% 10$ years subsequent to surgical excision (2). Bauer was the first to describe this entity in 1757 (3). Lipomas are the third most prevalent benign tumor of the large bowel, after hyperplastic and adenomatous polyps, and the incidence rate ranges from $0.2-4.5 \%$ worldwide $(1,4)$. These lesions are typically asymptomatic and are discovered incidentally during colonoscopy, surgery or autopsy (5). Gastroenterologists or radiologists are able to distinguish the benign nature of such tumors and characterize them as a lipoma; however, histopathological examination is required to reach a final diagnosis (6). In a small number of cases, lipomas may cause symptoms such as pseudo-obstruction, blunt abdominal pain, changes in daily defecation habits, rectal bleeding and constipation (6). In extremely rare cases, these lesions may also cause massive hemorrhage, full obstruction of the colon's lumen, intussusceptions and perforation, which results in acute abdomen and prolapse $(5,6)$. The size of lipomas may range from millimeters to centimeters in diameters, but symptoms occur only when their diameter exceeds $2 \mathrm{~cm}$ (7). The term 'giant lipoma' is used when its maximum diameter exceeds $5 \mathrm{~cm}$ (5). The current case describes a young Caucasian woman who presented to the Emergency Department of the University Hospital of Heraklion (Crete, Greece) due to a 1-month history of blunt pain in the lower abdomen and the presence of a solid mass, which prolapsed out of the anus following defecation. The present study described this rare case and reviewed the relevant literature in order to standardize the necessary procedures required for the diagnosis and treatment of these lesions.

\section{Case report}

In May 2013, a 27-year-old Caucasian woman was admitted to the Emergency Department of the University Hospital of 
A

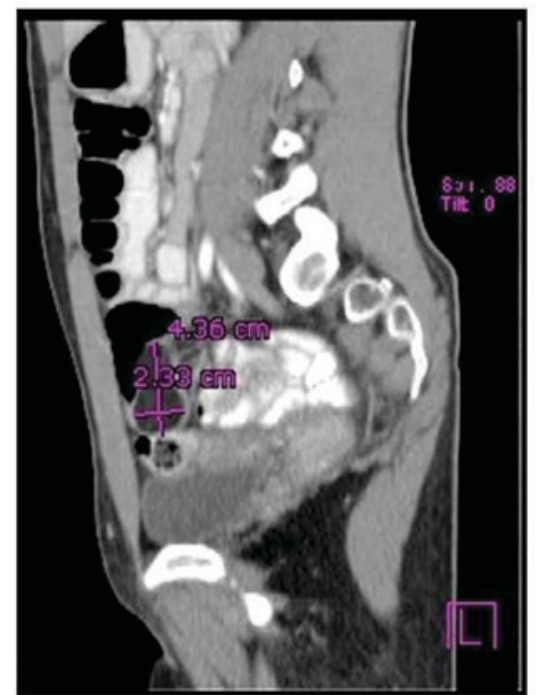

B

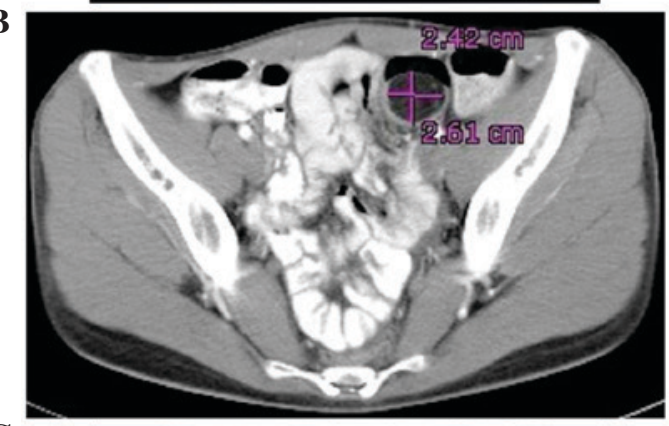

C

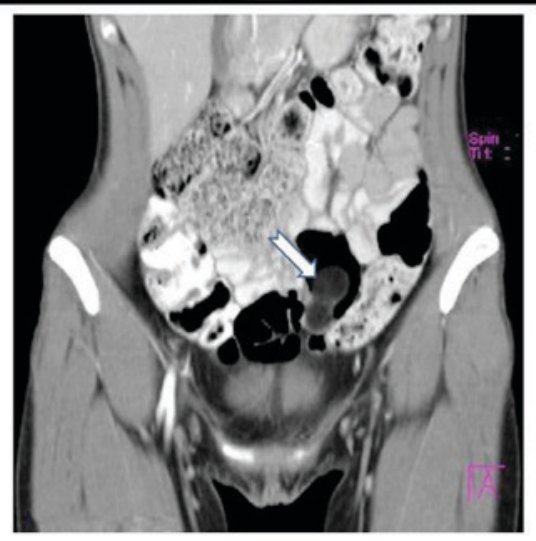

Figure 1. Contrast enhanced computed tomography showing the presence of a sharply defined mass in the sigmoid colon in (A) sagittal, (B) axial and (C) coronal views (white arrow, location of mass), without solid components, consistent with lipoma.

Heraklion (Crete, Greece). The patient presented with difficulty when defecating due to the presence of a solid mass, which prolapsed out of the anus following defecation for the previous month. The relevant medical history of the patient was unremarkable without disease, and clinical examination of the abdomen was unremarkable. Visual inspection of the anus and digital examination of the rectum did not identify any notable pathological findings. Therefore, the patient was asked to simulate the conditions of normal defecation with prolonged extrusion, and a solid, yellow mass protruding from the anal ring was observed.

The patient underwent an urgent colonoscopy, which revealed a suspicious polypoid lesion measuring $2.5 \mathrm{~cm}$ in diameter, which was located in the middle of the sigmoid colon

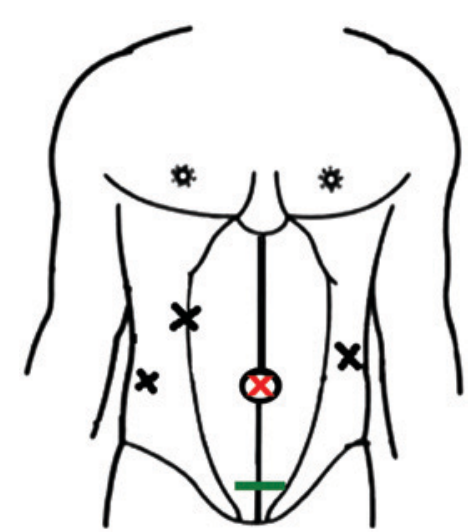

Figure 2. Trocar entry points. A small incision was made longitudinally at the umbilicus to establish an appropriate intra-abdominal pressure. A 12-mm trocar was inserted into the right-middle subcostal area and two 11-mm trocars were inserted into the frontal axillary line. A small $(4 \mathrm{~cm})$ horizontal incision was made in the middle line of the lower abdomen to remove the surgical specimen.

$\sim 12 \mathrm{~cm}$ above the anal ring, with a smooth surface and tissue fragility. Tissue samples were obtained and sent for histopathological analysis. Contrast-enhanced computed tomography (CT) of the abdomen was performed urgently and revealed the presence of a solid mass in the sigmoid colon (Fig. 1). Regional lymph nodes were not enlarged. Preoperative tests were completed, including blood tests, and cancer biomarkers [including carcinoembryonic antigen, $\alpha$-fetoprotein, carbohydrate antigen (CA)19-9, CA15-3 and CA125] were negative.

Following adequate intestinal preparation with laxatives, the patient underwent a laparoscopic segmental sigmoidectomy. The patient was placed in a traditional Lloyd-Davis position, with the left arm abduced and the right arm positioned along the body. During the procedure, the operating table was tilted towards the right and ranged between Trendelenburg and reverse Trendelenburg positions depending on the various operative steps.

Initially, a 3-mm incision was made under the umbilicus and a 12-mm trocar was inserted using the 'Hasson technique'. Insufflating carbon dioxide, pneumoperitoneum was established to an abdominal pressure of 12-14 $\mathrm{mmHg}$. Under direct vision with laparoscopic assistance, one $12-\mathrm{mm}$ trocar was inserted into the right-middle subcostal area and two $11-\mathrm{mm}$ trocars were inserted into the frontal axillary line in the bilateral regions of the abdomen (Fig. 2). Once the abdominal cavity was full of carbon dioxide at a preset intra-abdominal pressure, the sigmoid colon containing the lipoma was identified. This segment of the sigmoid colon was dissected with laparoscopic instruments (Ligasure ${ }^{\circledR}$ Dissector and Endo-GIA ${ }^{\mathrm{TM}} 30 \mathrm{~mm}$ ) using upward traction and a dissection from the medial to lateral side. Subsequently, a small horizontal incision of $4 \mathrm{~cm}$ was made in the midline of the lower abdomen, from which the surgical specimen was removed (Figs. 3 and 4). The small abdominal incision was sutured and pneumoperitoneum in the preset intra-abdominal pressure was re-established. Finally, an intracorporeal termino-terminal colorectal anastomosis was performed, with the use of a $28 \mathrm{~mm}$ circular stapler, without the use of a drain.

An intraoperative frozen section of the surgical specimen was not performed. Macroscopic inspection of the resected 


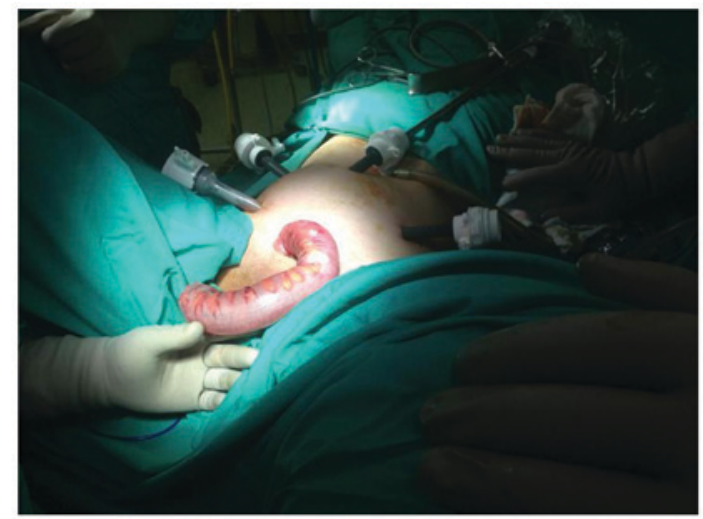

Figure 3. Sigmoid colon and the inferior region of the descending colon.

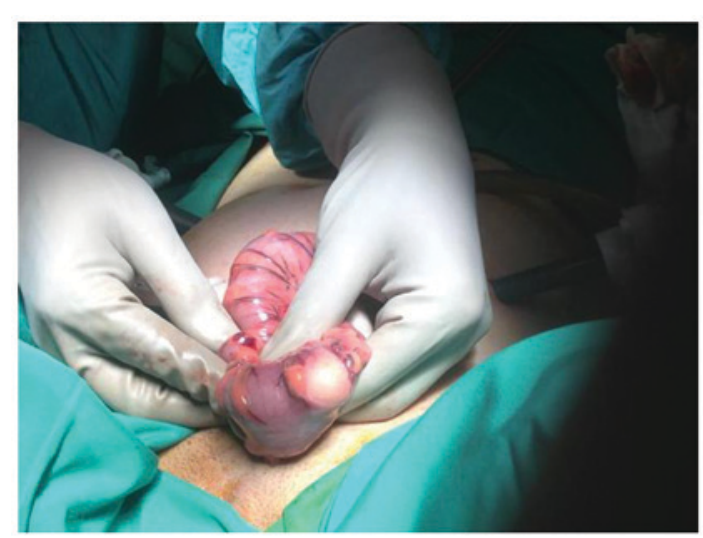

Figure 4. Lipoma in the sigmoid colon.

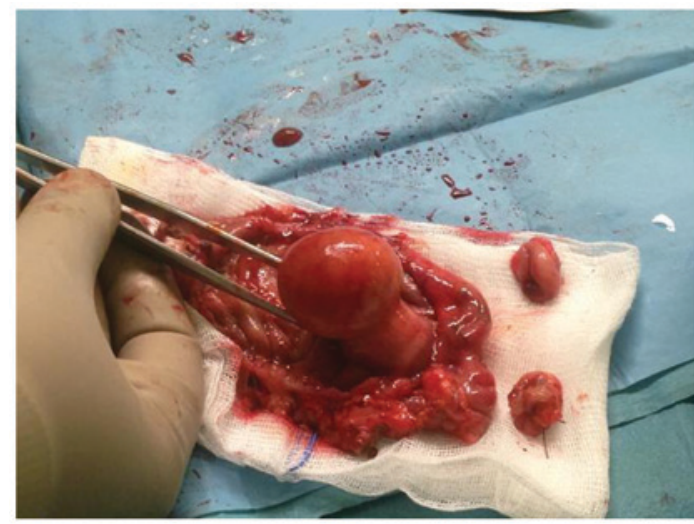

Figure 5. Surgical specimen with the lipoma in situ.

sigmoid colon confirmed the initial preoperative diagnosis (Fig. 5). The surgical specimen presented as a pedunculated lipoma that was shaped like a 'champagne bottle cork', and was covered in normal intestinal mucosa, with well-shaped, bright yellow parenchyma. The patient had a positive post-operative outcome without complications and was discharged on day 4 post-surgery in an optimal condition. Histopathological examination of the formalin-fixed surgical specimen revealed that the tumor was composed of mature adipose tissue compatible with a lipoma, and also exhibited regions of inflammatory changes in the colonic mucosa (Fig. 6). The overlying colonic

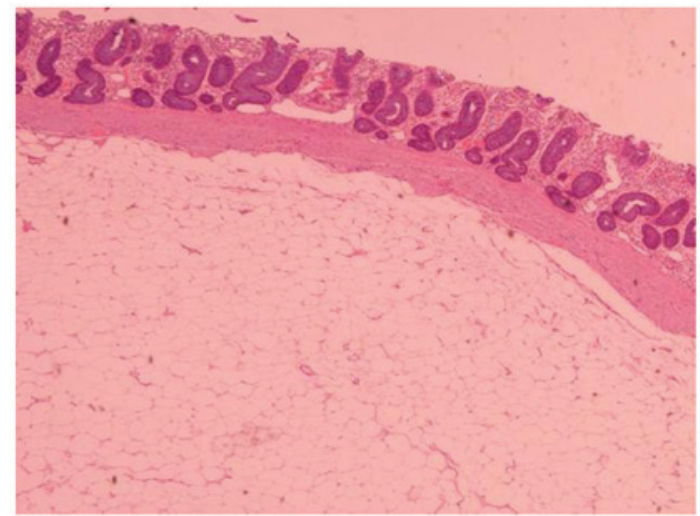

Figure 6. Histological examination of the surgical specimen demonstrating inflammatory changes to the colonic mucosa. The tumor consisted of mature adipose cells. The overlying colonic mucosa showed hyperplastic crypts with regenerative changes. In the lamina propria, mild inflammatory infiltration was observed (haematoxylin-eosin staining, 100x original magnification).

mucosa showed hyperplastic crypts with regenerative changes. In the lamina propria mild inflammatory infiltration was observed. Serial sections were obtained an embedded in paraffin. Hematoxylin and eosin staining was performed on $3-\mu \mathrm{m}$ sections, and the samples were observed under a light microscope (Nikon Eclipse E400; Nikon Corporation, Tokyo, Japan; magnification, x100. At 3 years post-surgery, the patient remains asymptomatic without any clinical evidence of recurrence. The patient undergoes planned follow-up appointments every 6 months, with blood tests (including cancer biomarkers) and CT scanning of the abdomen and chest once a year.

Written informed consent was obtained from the patient for publication of the present study and any accompanying images.

\section{Discussion}

Lipomas of the gastrointestinal tract are extremely rare (4). Esophageal lipomas are uncommon and account for $0.4 \%$ of all benign digestive tract neoplasms (8). Gastric lipomas are remarkably rare, benign tumors and are commonly located submucosally in the gastric antrum; these lesions represent $<5 \%$ of all lipomas of the gastrointestinal tract (9). Additionally, lipomas may also be identified accidentally within the small bowel due to intussusception and gastrointestinal bleeding (10).

Lipomas of the large intestine are uncommon, benign, adipose tumors that are typically asymptomatic and diagnosed accidentally during colonoscopy (3). Despite their rarity, they may occasionally induce symptoms such as lower abdominal pain, pseudo-obstruction of the colon, intussusceptions, hemorrhage and perforation (7). If they are localized to the sigmoid colon, the lipoma may prolapse out of the anus ring, as observed in the present case.

It may be challenging to obtain an accurate preoperative diagnosis of a colon lipoma. Nowadays, a wide range of imaging techniques are available that are able to help achieve a reliable diagnosis (11). Abdominal ultrasonography and abdominal radiography performed when the patient is in an upright and decubitus position do not typically aid the diagnosis of colon 
lipomas, due to their small size, the adipose consistency and the low distinctive ability of these imaging techniques (12). Abdominal double-contrast (intravenous and peros) CT may aid a reliable diagnosis, and are able to determine the size, position, morphology and proximity of the lipoma to adjacent organs and tissues (13). In addition, magnetic resonance imaging of the abdomen may help lipoma diagnosis, but does not appear to have superior diagnostic value compared with CT (14).

Endoscopic examination of the large colon serves an important role not only to achieve accurate diagnosis, but also when forming the final therapeutic plan for the patient. According to the literature, there is a theory that pedunculated lipomas with a diameter of $<2 \mathrm{~cm}$ may be removed by endoscopy without major complications (15).

Preoperative tests may include blood cell counts, hepatic and renal biochemical functional profiling, and evaluation of cancer markers (11). The adequate preoperative preparation of the patient is crucial for a positive surgical outcome. Solid preparation of the bowel with laxatives, and preoperative administration of antibiotics and prophylactic low-molecular-weight heparin for the prevention of deep vein thrombosis, are routine practices.

Once preoperative tests and preparation are completed, the surgeon must then decide whether to perform open surgery or use laparoscopic techniques.

With regards to open surgery, it is less likely that the surgeon may encounter intraoperative complications compared with laparoscopic techniques (16). However, there is an increase in the duration of intraoperative surgery and post-operative hospitalization time, and therefore, daily hospital charges are greater. Post-operative complications, including intra-abdominal abscesses, post-operative ileus, wound infection and respiratory failure, occur more often following open procedures. Post-operative chronic pain is less likely to occur in patients who undergo laparoscopic resection vs. those who undergo open laparotomy $(16,17)$. In the present case, the patient underwent a laparoscopic procedure and had an uneventful post-operative hospitalization.

It has been suggested that intraoperative frozen section of surgical specimens may provide an accurate diagnosis to guide the selection of surgical procedures (18). In the current case, histopathological examination of the surgical specimen was performed postoperatively, therefore further strategic planning of the surgical operation and intraoperative management of the patient was straightforward.

In conclusion, the present case highlights an extremely rare surgical entity and describes the process from diagnosis to surgical treatment (11). Colonoscopy and laparoscopy are considered as the best options for diagnosis and treatment, respectively, for patients with lipomas (15-17). The surgical excision of a sigmoid colon lipoma is a great challenge for surgeons. The correct pre-operative management, the appropriate radiological examinations and the right surgical method chosen by the surgeon can all lead to a successful result.
Surgical therapy, though challenging and demanding, may be the only mode of treatment that can offer these patients a return to a satisfactory level of function and quality of life. Informed consent is always necessary and ensures that the patient is fully aware of all the pros and cons. In conclusion, the present scientific report may assist surgeons worldwide with the correct decision making in similar cases.

\section{References}

1. Yaman İ, Derici H and Demirpolat G: Giant colon lipoma. Ulus Cerrahi Derg 31: 102-104, 2013.

2. Mnif L, Amouri A, Masmoudi MA, Mezghanni A, Gouiaa $\mathrm{N}$, Boudawara $\mathrm{T}$ and Tahri N: Giant lipoma of the transverse colon: A case report and review of the literature. Tunis Med 87: 398-402, 2009.

3. Ryan J, Martin JE and Pollock DJ: Fatty tumours of the large intestine: A clinicopathological review of 13 cases. Br J Surg 76: 793-796, 1989.

4. Vecchio R, Ferrara M, Mosca F, Ignoto A and Latteri F: Lipomas of the large bowel. Eur J Surg 162: 915-919, 1996.

5. Târcoveanu E, Chifan M, Veisa E, Epure O and Florea N: Colonic lipoma. Chirurgia (Bucur) 95: 353-357, 2000 (In Romanian).

6. Begos DG, Sandor A and Modlin IM: The diagnosis and management of adult intussusceptions. Am J Surg 173: 88-94, 1997.

7. Atmatzidis S, Chatzimavroudis G, Patsas A, Papaziogas B, Kapoulas S, Kalaitzis S, Ananiadis A, Makris J and Atmatzidis K: Pedunculated cecal lipoma causing colo-colonic intussusception: A rare case report. Case Rep Surg 2012: 279213, 2012.

8. Tsalis K, Antoniou N, Kalfadis S, Dimoulas A, Dagdilelis AK and Lazaridis C: Laparoscopic enucleation of a giant submucosal esophageal lipoma. Case report and literature review. Am J Case Rep 14: 179-183, 2013.

9. Ramdass MJ, Mathur S, Seetahal-Maraj P and Barrow S: Gastric lipoma presenting with massive upper gastrointestinal bleeding. Case Rep Emerg Med 2013: 506101, 2013.

10. Lucas LC, Fass R and Krouse RS: Laparoscopic resection of a small bowel lipoma with incidental intussusception. JSLS 14: 615-618, 2010.

11. Sourrouille I, Vilcot L, Honoré C, Coppola S, Terrier P, le Cesne A, Le Péchoux C and Bonvalot S: Algorithm for the surgical management of mesenchymal tumors of the perineum in adults. Dis Colon Rectum 58: 304-313, 2015.

12. Kuzmich S, Harvey CJ, Kuzmich T and Tan KL: Ultrasound detection of colonic polyps: Perspective. Br J Radiol 85: e1155-e1164, 2012.

13. Moussa OM, Tee M, Khan AU and Selvasekar CR: Computerized tomography providing definitive diagnosis of colonic lipoma: A case series. Surg Laparosc Endosc Percutan Tech 23: e232-e243, 2013.

14. Leufkens AM, Kwee TC, van den Bosch MA, Mali WP, Takahara T and Siersema PD: Diffusion-weighted MRI for the detection of colorectal polyps: Feasibility study. Magn Reson Imaging 31: 28-35, 2013.

15. Mummadi R and Raju GS: New endoscopic approaches to removing colonic lipomas. Gastroenterol Hepatol 3: 882-883, 2007.

16. Onder A, Benlice C, Church J, Kessler H and Gorgun E: Short-term outcomes of laparoscopic versus open total colectomy with ileorectal anastomosis: a case-matched analysis from a nationwide database. Tech Coloproctol 20: 767-773, 2016.

17. Stormark K, Søreide K, Søreide JA, Kvaløy JT, Pfeffer F, Eriksen MT, Nedreb $\varnothing$ BS and Kørner H: Nationwide implementation of laparoscopic surgery for colon cancer: Short-term outcomes and long-term survival in a population-based cohort. Surg Endosc 30: 4853-4864, 2016.

18. Khoury W, Abboud W, Hershkovitz D and Duek SD: Frozen section examination may facilitate reconstructive surgery for mid and low rectal cancer. J Surg Oncol 110: 997-1001, 2014. 